Cite this: DOI: $10.1039 / \mathrm{c0xx} 00000 x$

FULL PAPER

www.rsc.org/xxxxxx

This is the peer reviewed version of the following article:

T. Braschler, S. Wu, F. Wildhaber, S.A. Bencherif, and D.J. Mooney, Soft nanofluidics governing minority ion exclusion in charged hydrogels. Soft Matter, 2015. 11(20): p. 4081-90.

, which has been published in final form at

https://dx.doi.org/10.1039/c5sm00705d

10 . 


\title{
Soft nanofluidics governing minority ion exclusion in charged hydrogels
}

\author{
${ }_{5}$ Braschler Thomas, ${ }^{* a, b, c}$ Wu Songmei, ${ }^{b}$ Wildhaber Fabien $^{b}$, Bencherif Sidi A ${ }^{a, c, d}$, Mooney David $\mathbf{J}^{c, e}$ \\ Received (in $X X X, X X X)$ Xth $X X X X X X X X X 20 X X$, Accepted Xth XXXXXXXXX 20XX \\ DOI: $10.1039 / \mathrm{b000000x}$
}

${ }^{a}$ School of Engineering and Applied Sciences, Mooney lab, 60 Oxford Street, Harvard University, Cambridge, MA 02138, USA

${ }^{b}$ School of Engineering, LMIS4, Station 17, 1015 Lausanne, Switzerland

${ }_{10}{ }^{c}$ Wyss Institute for Biologically Inspired Engineering at Harvard University, 3 Blackfan Circle, Boston, MA 02115, USA

${ }^{d}$ UTC CNRS UMR 7338, Biomechanics and Bioengineering (BMBI), University of Technology of Compiègne, BP 20529,

Rue Personne de Roberval, 60205 Compiègne, France

${ }^{e}$ School of Engineering and Applied Sciences, 319 Pierce Hall, 29 Oxford Street, Harvard University, Cambridge, MA 02138, USA

We investigate ionic partition of negatively charged molecular probes into also negatively charged, 15 covalently crosslinked alginate hydrogels. The aim is to delimit the domain of validity of the major nanoelectrostatic models, and in particular to assess the influence of hydrogel chain mobility on ionic partition. We find that the widely used Gibbs-Donnan model greatly overestimates exclusion of the co-ion probes used. For low molecular weight probes, a much better fit is obtained by taking into account the electrostatics in the nanometric gel pores by means of the Poisson-Boltzmann framework; the fit is

20 improved slightly when taking into account alginate chain mobility. For high molecular weight probes, we find it essential to take into account local gel deformation due to electrostatic repulsion between the flexible gel strands and the probe. This is achieved by combining Poisson-Boltzmann simulations with heterogeneous pore size distribution given by the Ogston model, or more simply and precisely, by applying a semi-empirical scaling law involving the ratio between Debye length and pore size.

\section{${ }_{25}$ Introduction}

Hydrogels arguably represent the quickest, most accessible, and cheapest method to construct an extended nanochannel network. Indeed, they naturally consist of a nano-meshwork of polymer strands with water-filled pores between them. ${ }^{1}$ This nanostructure 30 affords molecular sieving and redistribution effects for solutes percolating the hydrogel, underpinning a wide range of applications, including chromatography, ${ }^{2}$ drug delivery, ${ }^{3}$ gel electrophoresis ${ }^{4}$ and the distribution of biomolecules in the human body. ${ }^{1}$ While molecular partition and transport in neutral ${ }_{35}$ hydrogels has been relatively well characterized, ${ }^{5}$ the theoretical understanding of charged hydrogels has remained more limited, ${ }^{6}$ despite growing application in drug delivery and tissue engineering. This is in part due to complexity of the interaction between nanostructure, molecular dynamics and electrostatics, 40 and in part because only recently nanoelectrical models have begun to be thoroughly tested in manmade, precisely controlled nanostructures. $^{7-9}$ We compare here an established phenomenological ionic distribution model, namely the Gibbs-
Donnan model, ${ }^{10,11}$ to more detailed nanoelectrical analysis at the 45 level of the hydrogel pore size, by means of the PoissonBoltzmann equation. ${ }^{10,11}$ Indeed, Poisson-Boltzmann models are widely used in the analysis of free polyelectrolyte solutions, known to strongly deviate from the Gibbs-Donnan theory. ${ }^{12,13}$

We also ask how the molecular mobility of the polymer 50 strands making up the hydrogel affects molecular redistribution in charged hydrogels. We use quantitative analysis of ionic exclusion of negatively charged solutes from also highly negatively charged, covalently crosslinked model alginate hydrogels in order to delimit the conditions of validity of the ${ }_{55}$ different models, and to assess the influence of chain mobility and therefore apparent charge distribution on ionic partition.

The question of ionic partition into charged hydrogels is an old one. The traditional approach is to apply the Gibbs-Donnan equilibrium theory. ${ }^{14}$ Its main, and most critical, assumption is a ${ }_{60}$ homogeneous fixed space charge distribution. ${ }^{15}$ This assumption is realistic if either the actual pore diameter is below the Debye electrostatic screening length, ${ }^{12,} 15$ or potentially if other 
mechanisms such as high chain mobility can recover an apparently more homogeneous space charge density despite larger nominal pore size. The Debye length for biologically and chemically relevant millimolar to molar free salt concentrations is ${ }_{5}$ below $10 \mathrm{~nm}$. While some hydrogels, namely polyacrylates with a very high monomer concentration do have such small pores, ${ }^{16}$ the diameter in typical protein or carbohydrate hydrogels is in the range of $10 \mathrm{~nm}$ to $1 \mu \mathrm{m}$ and sometimes beyond. ${ }^{17-20}$

We investigate here how ionic partition in alginate gels with a ${ }_{10}$ pore size in the range of $10-40 \mathrm{~nm}$ should be modeled : Either by the use of the Gibbs-Donnan equilibrium, assuming homogeneous space distribution due to a hypothetical infinitely high chain mobility? Or by the use of the Poisson-Boltzmann equations, assuming a static gel structure? Or even by an 15 intermediate model allowing for partial gel strand mobility?

While in the Gibbs-Donnan model, the fixed space charge is considered to be fully spread out, the opposite limiting case is the assumption of a completely static hydrogel, where fixed space charge is concentrated in the small solid fraction occupied by the

20 hydrogel polymer strands. We refer to this case as " solid nanochannel model ", since it corresponds to the theory commonly employed in manmade nanofluidic channels. ${ }^{7}$ When allowing for partial polymer strand mobility, a larger effective volume fraction is occupied by the fixed space charge, we refer to 25 this case as « soft hydrogel model. »

We further investigate the partitioning of a macromolecular, highly negatively charged probe into the covalent alginate gels, in order to better understand the relationship between ionic exclusion effects for macromolecules versus small molecules.

30 Indeed, while electrostatic interaction is considered to be of prime importance of small molecules, the pore size distribution according to the Ogston model $^{5}$ is reported to be of main importance for the partitioning of macromolecules into hydrogels, including charged gels. ${ }^{21}$ It is an intriguing possibility to assume

35 that due to the hydrogel network mobility, the gel can make room for macromolecules, and that the Ogston distribution may arise dynamically rather than being statically present. In that case, using the Ogston model for partitioning of small ions should not improve, or even deteriorate the fit between experimental 40 partitioning data and theory, while it should dramatically improve the fit for macromolecular probes.

\section{Theory}

We model charged hydrogels as one-dimensional, periodic arrays of fixed space charge, the local concentration of charged residues ${ }_{45}$ being given by the periodically varying function $c_{\text {fixed }}(x)$. The use of a periodic, evenly space lattice as a model for the charged hydrogel reflects the idea that high electrostatic repulsion between the chains should maximize and homogenize the spacing between the polymer strands making up the gel ${ }^{12}$. The periodic 50 space charge distribution gives rise to a periodic electrical potential distribution $\Phi(x)$, which is obtained by the solution to the Poisson-Boltzmann equation ${ }^{10,11,22}$ :

$$
\frac{\mathrm{d}^{2} \Phi}{\mathrm{d} x^{2}}=\frac{N_{\mathrm{A}} q_{\mathrm{e}}}{\varepsilon_{\mathrm{r}} \varepsilon_{0}} \cdot\left[c_{0}\left(e^{\Phi(x) / V_{\mathrm{th}}}-e^{-\Phi(x) / V_{\mathrm{th}}}\right)-c_{\text {fixed }}(x)^{-}\right.
$$

${ }_{55}$ where $c_{0}$ is the bulk free monovalent salt concentration,
$V_{\mathrm{th}}=k_{\mathrm{B}} T / q_{\mathrm{e}}=25.3 \mathrm{mV}$ the thermal voltage at a temperature of $T=293 \mathrm{~K}, q_{\mathrm{e}}=1.6 \cdot 10^{-19} \mathrm{C}$ the elementary charge, $\mathrm{N}_{\mathrm{A}}=6.022 \cdot 10^{23}$ $\mathrm{mol}^{-1}$ Avogadro's number, $\varepsilon_{0}=8.85 \cdot 10^{-12} \mathrm{Nm}^{2} / \mathrm{C}^{2}$ the vacuum permittivity, $\varepsilon_{\mathrm{r}}=78$ the relative permittivity and $k_{\mathrm{B}}=1.38 \cdot 10^{-23} \mathrm{~J} / \mathrm{K}$ ${ }_{60}$ the Boltzmann constant, and $c_{\text {fixed }}$ the local fixed space charge density associated with the hydrogel polymer strands. The periodicity of the structure, together with global electroneutrality, imposes that:

$$
\frac{\mathrm{d} \Phi}{\mathrm{d} x}=0 \text { for } x=0 \text { and } x=r_{\text {pore }}
$$

${ }_{65}$ where $r_{\text {pore }}$ is the pore radius. The two boundary conditions described by equation (2) uniquely define the solution $\Phi(\mathrm{x})$ to the ordinary, second-order differential equation given by equation (1). The fundamental behavior of the solutions to eq. 1 and 2 depends on the ratio of the pore radius $r_{\text {pore }}$ to the Debye length ${ }_{70} l_{\text {Debye }}:{ }^{15}$

$$
\lambda=\frac{r_{\text {pore }}}{l_{\text {Debye }}}
$$

where the Debye length $l_{\text {Debye }}$ is given by: ${ }^{23}$

$$
l_{\text {Debye }}=\sqrt{\frac{\varepsilon_{0} \varepsilon_{r} k_{B} T}{2 N_{A} q_{e}^{2} c_{0}}} \approx \frac{0.3 \mathrm{~nm}}{\sqrt{c_{0}[\mathrm{M}]}}
$$

The fixed space charge distribution depends on the volume 75 fraction $v$ accessible to the polymer strands, and can be expressed in a simplified manner as follows:

$$
\begin{aligned}
& c_{\text {fixed }}(x)=\frac{\bar{c}_{\text {fixed }}}{v} \text { for } 0 \leq x \leq v \cdot r_{\text {pore }} \text { and } \\
& c_{\text {fixed }}(x)=0 \text { for } v \cdot r_{\text {pore }}<x \leq r_{\text {pore }}
\end{aligned}
$$

where $\bar{c}_{\text {fixed }}$ is the overall average concentration of fixed charges ${ }_{80}$ linked to the polymer. We use equation 5 generically, but use different expressions for the accessible volume fraction $v$, reflecting the model assumptions.

For the Gibbs-Donnan model, the space charge is assumed to be distributed homogeneously, such that $v=v_{\text {Donnan }}$ given by:

${ }_{85} \quad v_{\text {Donnan }}=1$

In the complete absence of chain mobility (« solid nanochannel» model), the solid volume fraction occupied by the polymer strands $v=v_{\text {solid }}$ is given by the dry volume fraction:

$$
v_{\text {solid }}=\frac{c_{m}}{\rho}
$$

90 with $c_{m}$ being the polymer concentration in units of mass per volume and $\rho$ the dry polymer density.

In the case of limited polymer strand mobility ("soft hydrogel" model), we assume the accessible volume fraction $v=v_{\text {soft }}$ to reflect the space that the polymer strands can explore due to ${ }_{95}$ thermal agitation. For the highly charged, relatively soft gels used here, electrostatic interchain repulsion is considered to be the 
main restraining force, such that we expect the accessible space to be a function of the ratio of the electrostatic screening length $l_{\text {Debye }}$ and the pore size. We propose to estimate $v_{\text {soft }}$ as follows:

$$
v_{\text {soft }}=\left(1+\alpha \frac{l_{\text {Debye }}}{r_{\text {pore }}}\right)^{-1}=\frac{\lambda}{\lambda+\alpha}
$$

5 where $\alpha$ is an empirical constant reflecting inter-chain repulsion. Expression (6-C) is chosen semi-empirically such as to reflect repulsion from a central area of $\alpha \cdot l_{\text {Debye }}$ for $\alpha \cdot l_{\text {Debye }}<<r_{\text {pore }}$, while satisfying $v_{\text {soft }} \rightarrow 0$ for $\lambda=r_{\text {pore }} / l_{\text {Debye }} \rightarrow 0$ and $v_{\text {soft }} \rightarrow 1$ for $\lambda \rightarrow \infty$. Hence, it directly links the effective chain repulsion to the 10 fundamental dimensionless ratio $\lambda$ of pore radius to Debye length.
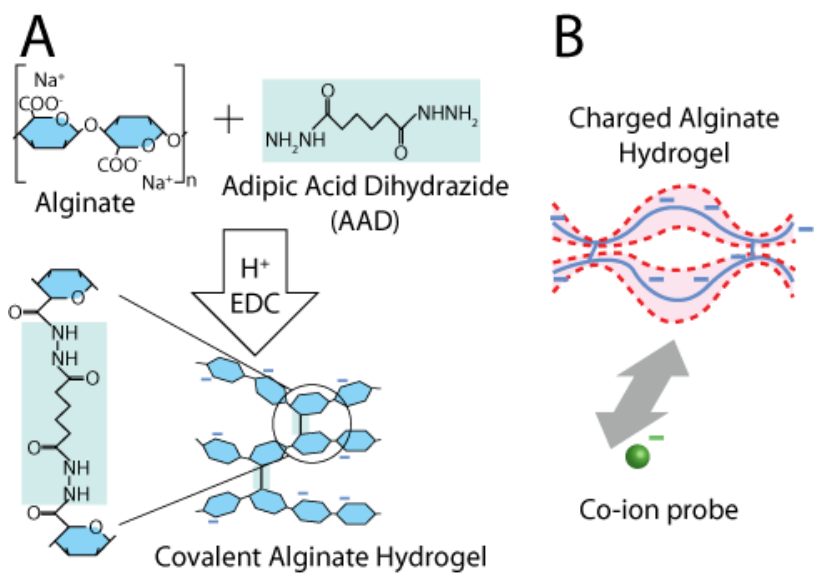

Co-ion probe

Figure 1. Investigation of ionic partition into negatively charged alginate 15 hydrogels. A) Chemistry used for covalent crosslinking of alginate Adipic acid dihydrazide is used to provide covalent crosslinks between carboxyl groups of the alginate chain. To do so, the carboxylgroups are activated by a carbodiimide (EDC), followed by condensation with the amino groups of the adipic acid dihydrazide ${ }^{24,25}$. B) Ionic partition. We 20 investigate theoretically and experimentally the partition of tracer substances into the alginate hydrogels at various ionic strengths. On the theoretical level, the hydrogel can be modeled by fixed negative space charges (blue) with limited thermal mobility (shaded areas). We are mostly interested in the partition of negatively charged co-ion probes in 25 the gel.

The inhomogeneous distribution of the space charge for the solid nanochannel and soft hydrogel model (eq. 6-B respectively 6-C) requires numerical integration of equations 1 and 2 to obtain a numerical solution for $\Phi(x)$ for the fixed charge densities 30 specified by equation 5 in conjunction with $6-\mathrm{B}$ or $6-\mathrm{C}$. On the other hand, under the Gibbs-Donnan approximation (equation 6A), the solution is particularly simple ${ }^{14}$ :

$$
\Phi(x)=\text { const }=\Phi_{\text {Donnan }}=V_{\text {th }} \cdot \ln \left[\frac{\bar{c}_{\text {fixed }}}{2 c_{0}}+\sqrt{1+\left(\frac{\bar{c}_{\text {fixed }}}{2 c_{0}}\right)^{2}}\right] \text { (7) }
$$

Whatever the model used, the macroscopically observable ${ }_{35}$ partition coefficients for tracer ions are obtained by integration of the local concentrations:

$$
K^{n \pm}=\frac{1}{r_{\text {pore }}} \int_{0}^{r_{\text {pore }}} \mathrm{e}^{\mp n \Phi(x) / V_{\text {th }}} \mathrm{d} x
$$

where $n$ is the number of charges carried by the tracer ion under consideration. In the Gibbs-Donnan limit, this yields a simple 40 analytical expression:

$$
K_{\text {Donnan }}^{n \pm}=\mathrm{e}^{\mp n \Phi_{\text {Donnan }} / V_{\text {th }}}
$$

The solid nanochannel model corresponds to a well-studied case of the Poisson-Boltzmann equation, namely the case of free electrolyte next to a sharply defined charged boundary ${ }^{7}, 12,13$. ${ }_{45}$ Based on the developments by Overbeek ${ }^{12}$ for dilute and concentrated polyelectrolyte solutions, we can estimate the partition coefficient in the solid nanochannel model as follows:

$$
K_{\text {solid }}^{ \pm n}=\frac{\int_{\Phi_{m} / V_{t h}}^{\Phi_{0} / V_{t h}} e^{\mp n y} \cdot\left(\cosh (y)-\cosh \left(\Phi_{m} / V_{t h}\right)\right)^{-\frac{1}{2}} \mathrm{~d} y}{\int_{\Phi_{0} / V_{t h}}^{V_{t h}}\left(\cosh (y)-\cosh \left(\Phi_{m} / V_{t h}\right)\right)^{-\frac{1}{2}} \mathrm{~d} y}
$$

where $\Phi_{\mathrm{m}}$ is the potential at the channel midline (i.e. $\left.\Phi\left(x=r_{\text {pore }}\right)\right)$ and $\Phi_{0}$ the potential near the alginate fibers (i.e. $\Phi(x=0)$ ). Eq. 10 is derived in Electronic Supplementary Information 6 (Fig. S31, 55 eq. S63), along with analytical expressions for $\Phi_{\mathrm{m}}$ and $\Phi_{0}$ (Fig. S31, S35, and eq. S34, S50, S58 in Electronic Supplementary Information 6). Numerical integration of eq. 8 is generally necessary for the soft hydrogel model and also if an exact solution is desired for the solid nanochannel model.

${ }_{60} \mathrm{We}$ also investigate the partition of a highly charged macromolecular probe into the hydrogels. For this, we use soluble fluorescently labeled alginate as a probe. We expect the gel to be locally deformed as a response to the large size and charge of the macromolecular probe, leading to a significant ${ }_{65}$ decrease of electrostatic repulsion energy. This effect is naturally accessible to the soft nanochannel model. Indeed, assuming the limitation of the freedom of movement of the highly charged macromolecular probe to be similar in nature to the limitation of movement of the gel strands, the partition coefficient for the 70 macromolecular probe should be given by:

$$
K=\left(1+\beta \frac{l_{\text {Debye }}}{r_{\text {pore }}}\right)^{-1}=\frac{\lambda}{\lambda+\beta}
$$

where $\beta$ is an empirical constant. Eq. 11 is analogous to eq. $6-\mathrm{C}$, outlining again the fundamental importance of $\lambda$ in the context of electrostatic interaction with charged hydrogels. Eq. 11 should be 75 valid in the limit of purely electrostatic repulsion. For less densely charged macromolecules such as typical soluble proteins, spatial constraints would need to be taken into account as well. ${ }^{21}$

It is also possible to model the partition of a macromolecular, highly charged probe in the framework of the solid nanochannel 80 model, although a number of experimental and theoretical complications need to be taken into account if realistic predictions are to be obtained. A first issue is local pore size heterogeneity, which we consider to arise as a response to the presence of the probe rather than being statically present. The ${ }_{85}$ Ogston model provides the distribution of the pore radius if its mean $r_{0}$ is known ${ }^{5}$, such that the overall partition coefficient 
$K_{\text {overall }}$ is then obtained by averaging (Details are given in Electronic Supplementary Information 1):

$$
\begin{aligned}
& K_{\text {overall }}=\int_{r_{\text {pore }}=0}^{r_{\text {pore }}=\infty} K\left(r_{\text {pore }}\right)\left(\frac{\mathrm{d} P}{\mathrm{~d} r_{\text {pore }}}\right) \cdot \mathrm{d} r_{\text {pore }} \text { with } \\
& \frac{\mathrm{d} P}{\mathrm{~d} r_{\text {pore }}}=2 \Gamma\left(\frac{3}{2}\right)^{2} \cdot \frac{r_{\text {pore }}}{r_{0}^{2}} \cdot \exp \left[-\left(\Gamma\left(\frac{3}{2}\right) \cdot \frac{r_{\text {pore }}}{r_{0}}\right)^{2}\right]
\end{aligned}
$$

5 where $\Gamma$ is the gamma function.
A

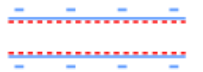

Solid nanochannel
B

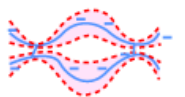

Soft hydrogel
C

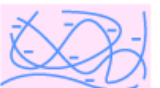

Gibbs-Donnan

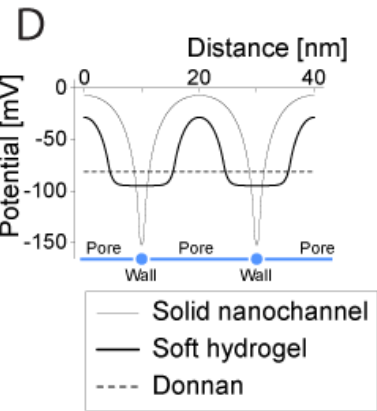

E

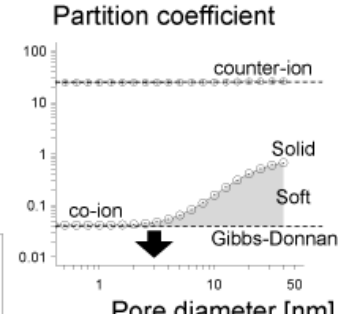

Pore diameter $[\mathrm{nm}]$

Figure 2. Theoretical frameworks for ion partition in charged hydrogels Static nanostructure in the solid nanochannel model (A), limited mobility in the soft hydrogel model (B) and fully homogeneous space charge in the

${ }_{10}$ Gibbs-Donnan theory (C). Fig. 2D shows the electric potential distribution at $10 \mathrm{mM}$ salt strength in a $1 \mathrm{D}$ model with $20 \mathrm{~nm}$ pore size for the three models, the soft nanochannel model corresponding to an effective wall volume fraction of 0.6. E) Expected partition coefficients for a singly charged co-ion (single negative charge), a singly charged 15 counter-ion (single positive charge) as a function of the spacing of the space charge regions in the array. For the solid nanochannel simulations, the fixed space charge is assumed to be concentrated in periodic patches occupying $5 \%$ of the available space, and is assumed to have a local concentration of $5 \mathrm{M}$ negative residues; overall, this gives to a mean space 20 charge of $250 \mathrm{mM}=-0.24 \mathrm{C} / \mathrm{m}^{3}$. The structure was assumed to be at thermodynamic equilibrium with a monovalent salt solution of $10 \mathrm{mM}$, giving rise to a Debye length of $3 \mathrm{~nm}$ (black arrow in Fig. 2E). The soft nanochannel model gives partition coefficients intermediate between the Donnan model and the solid nanochannel model (grey area).

${ }_{25} \mathrm{~A}$ second theoretical issue arising with highly charged macromolecules is Manning counter-ion condensation and shielding by the mobile charges ${ }^{13}$, both effects leading to lowering of the effective electrostatic repulsion. By explicit calculation of the electrostatic repulsion energy in the Poisson-

30 Boltzmann framework ${ }^{13,21}$, we take these effects into account; we calculate an effective charge density that lies in the range of $10 \%$ $30 \%$ of the nominal charge density for the relevant experimental conditions (Details are given in Electronic Supplementary Information 1).

35 Finally, the probe used is polydisperse, i.e. it has a distribution of molecular weights rather than a single defined strand length. Given that the partition coefficients will be dependent on the molecular weight, we obtain the overall partition coefficient by integration over the different molecular weights as determined by

40 gel permeation chromatography GPC (details in Electronic Supplementary Information 1).

In order to compare the partition phenomena observed for small ions and polyelectrolytes in charged covalent alginate hydrogels to theoretical predictions, we finally need to estimate 45 the pore size of the hydrogels. We do so by estimating the macroscopic hydraulic conductivity of the gels. We estimate the hydraulic conductivity from stress relaxation as quantified by mechanical testing, as shown in Figure 3A. It is indeed wellknown that in covalent hydrogels, stress relaxation primarily so reflects water migration ${ }^{24}$. Since in addition, pore size is known to be related to the hydraulic conductivity of hydrogels ${ }^{26}$ the time constant of the stress relaxation along with the compressive Young modulus can be used to estimate the pore radius:

$$
r_{\text {pore }}=R_{\text {sample }} \cdot \sqrt{\frac{8 \eta}{E \tau}}
$$

${ }_{55}$ where $R_{\text {sample }}$ is the macroscopic radius of the disk used for compression testing, $\eta$ the viscosity of the pore medium (here, water, with $\eta=10^{-3} \mathrm{~Pa}$ ), $E$ the gel's compressive Young modulus, and $\tau$ the relaxation time. The details of the derivation of eq. 13 as well as the fitting procedure for obtaining $\tau$ for disk-shaped 60 samples are outlined in Electronic Supplementary Information 2.

\section{Results}

In this report, we investigate the partition of negatively charged probes into also negatively charged model hydrogels. For this, we synthesized covalently crosslinked alginate hydrogels by well${ }_{65}$ established carbodiimide chemistry ${ }^{24,25}$, with water-soluble carbodiimide (EDC) as the activator, and the di-amine adipic dihydrazide as the crosslinker (Fig. 1A). The gels set within a few minutes, allowing for convenient molding into arbitrary shapes before gel formation.

70 We use these gels as model hydrogels to address the fundamental question of the appropriate choice of the theoretical model for ionic partition: does the chain mobility in hydrogels lead to an effective space charge distribution that is sufficiently homogeneous to apply the simple Gibbs-Donnan equilibrium 75 theory? Or is it more appropriate to consider a solid nanochannel model taking into account the hydrogel's nanostructure consisting of polymer chains and water-filled channels between them? Or do we need a finer, intermediate soft hydrogel model allowing for partial chain mobility?

${ }_{80}$ To address these questions, we synthesized the covalently crosslinked alginate hydrogels at different gel concentrations. We then investigated the partition of negatively charged probe substances into these gels (Fig. 1B) under different ionic force conditions ( $\mathrm{KCl}$ concentrations). We compared the experimental 85 observations to three theoretical models, namely the solid nanochannel model, the soft hydrogel model, and the GibbsDonnan model (Fig. 2). The solid nanochannel model covers the extreme limit of essentially immobile polymer strands making up the hydrogel. In this case, the entire fixed negative charge 90 associated with the alginate molecules is concentrated in a very small volume fraction given by the dry fraction of polymer present (Fig. 2A). The soft hydrogel model allows for partial mobility of the polymer strands, such that the fixed negative 
charge linked to the polymer molecules appears partially smeared out (Fig. 2B). The Gibbs-Donnan model finally assumes a completely homogeneous space charge distribution (Fig. 2C).

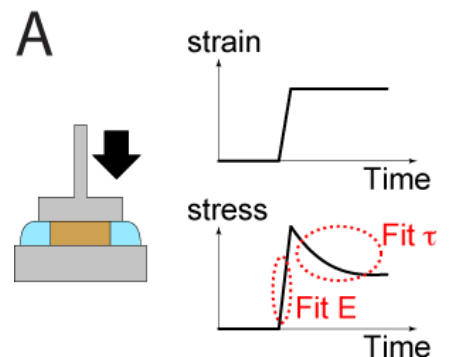

Pore size from $\tau$ and $\mathrm{E}$
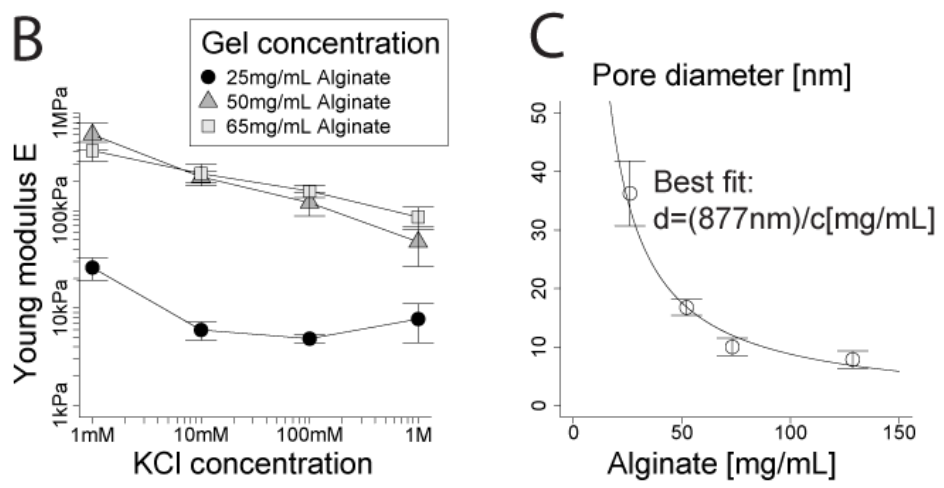

Figure 3. Pore size characterization by mechanical compression. A) Stress relaxation is monitored by rapidly compressing a hydrogel sample, and then 15 monitoring the stress necessary to maintain a given strain over time. From the compression phase, the Young modulus $E$ can be obtained, while the stress relaxation can be described by the stress relaxation time constant $\tau$. Modified from $\mathrm{ref}^{24} \mathrm{~B}$ ) Compressive Young modulus as a function of alginate concentration and ionic force. C) Pore diameter as a function of alginate concentration, measured in PBS.

In all three cases, we can model the hydrogel by a 1D model with a simplified periodic structure ${ }^{15}$, consisting of alternating polymer 20 " walls » and aqueous pore space (Fig 2D). The local electric potential is assumed to follow the Poisson-Boltzmann equation (eq. 1). Using suitable boundary conditions (eq. 2), along with the characteristic fixed charge distribution specific to each of the three models (eq. 5, and 6-A, 6-B or 6-C as a function of the 25 model), the local electric potential distribution in the pores is obtained (Fig. 2D).

As expected ${ }^{27}$, the Gibbs-Donnan model gives rise to a homogeneous electric potential distribution, while the solid nanochannel and the soft hydrogel model both give rise to a more 30 negative electric potential in the vicinity of the polymer chains, and a more nearly neutral electric potential towards the midline of the aqueous pores between the polymer strands. The ionic partition coefficients of a given positively or negative charged ion can then be evaluated by integration of its local concentration (eq.

358 ). The simulation was carried out for different pore sizes, such that the partition coefficients for the different models can be traced as a function of the pore size. This was done in Fig. 2E. The results indicate firstly that the partition coefficient of singly charged counter-ions (positively charged ions for the negatively

40 charged gel used here) is little affected by the choice of the underlying theoretical model (upper horizontal line in Fig. 2E, all three models are superimposed). This essentially reflects the fact that under the chosen conditions, the counter-ions are the majority mobile ionic species in the gel, and as such they provide 45 for electroneutrality and thus must essentially balance the negative charge of the alginate molecules ${ }^{12}$. The result also means that in order to probe the influence of pore structure and dynamics on the ionic partition, we need to investigate the partition of co-ions (negatively charged in our case) rather than 50 the partition of counter-ions, for which the models essentially give identical results.

The exclusion of the co-ions becomes increasingly less efficient for the solid nanochannel model than for the Gibbs-Donnan model for pore sizes exceeding the Debye length (Fig. 2E). The ${ }_{55}$ reason for this can be seen in Fig. 2D: the electrostatic shielding of the central area of the pores leads to a near neutral potential, associated with ionic composition near bulk far from the channel walls. While quantitatively negligible for the otherwise highly concentrated counter-ions, these shielded areas give rise to much ${ }_{60}$ higher partition coefficients for the co-ions than expected based on the homogeneous charge and electric potential distribution assumed by the Gibbs-Donnan theory.

The soft hydrogel model is intermediate between the GibbsDonnan and solid nanochannel model (grey area in Fig. 2E). ${ }_{65}$ Indeed, the increased polymer chain mobility and thus apparent « smear out " gives rise to a smoother electric potential distribution (Fig. 2D), and thus also more efficient co-ion exclusion at larger pore size. The exact value of the partition coefficient will however depend on the chain mobility parameter $\alpha$ (eq. 6-C).

70 To compare experimentally measured partition coefficients to the theoretical predictions, an estimation of the average pore diameter is needed for the solid nanochannel and soft hydrogel models. The pore diameter has a dramatic influence on water migration under pressure gradients in porous media in general ${ }^{28}$, 75 and in hydrogels in particular ${ }^{26}$. In covalently crosslinked alginate hydrogels, stress relaxation is known to be mainly related to water migration in the hydrogel meshwork ${ }^{24}$. Hence, we use quantification of stress relaxation by mechanical compression testing to evaluate the pore diameter (Fig. 3A). First, we used a ${ }_{80}$ rapid compression phase (seconds) to determine the elastic modulus $\mathrm{E}$ of the gels. Fig 3B shows the limiting elastic moduli for small deformations as a function of ionic force and alginate concentration. It can be seen that the elastic modulus increases with lower ionic force for most cases, except for the highest ionic 85 forces and lowest alginate concentration. This is indicative of a dominating effect of high electrostatic interchain repulsion, which increases with decreasing ionic force. We then quantified stress relaxation on the compressed gels by keeping the strain constant, while measuring the decreasing elastic force and therefore stress 
over up to $3 \mathrm{~h}$ following the initial compression. During this time, pore water is squeezed out from the gels, and the elastic force decreases as pore pressure approaches ambient pressure. The pore diameter can then be calculated from the compressive Young 5 modulus and the stress relaxation time constant (eq. 13, further details in Electronic Supplementary Information 2). The results are shown for gels of varying alginate concentration equilibrated in PBS (Fig. 3C). The pore size is measured to be inversely proportional to the alginate concentration. 


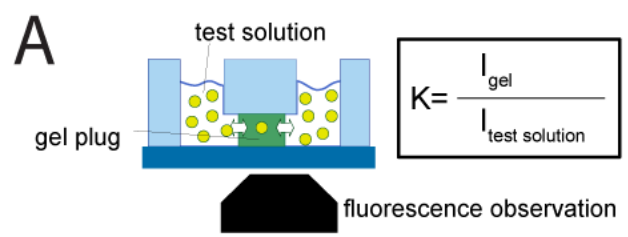

Legend Fig. 4B-4D
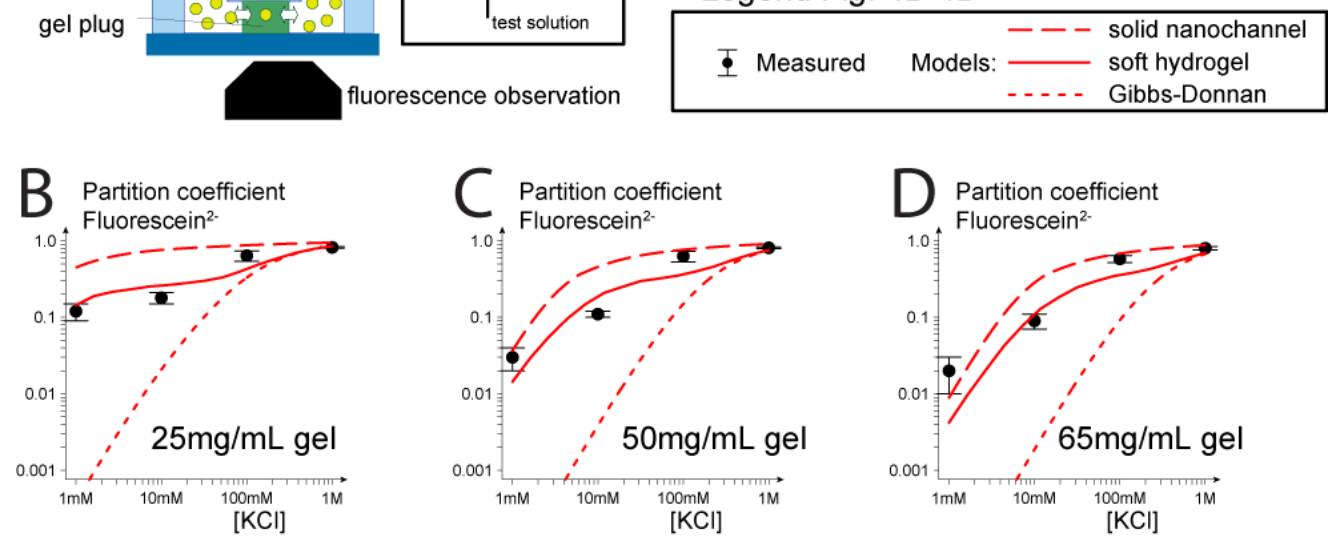

Figure 4. Partition coefficients for fluorescein dianion. Figure 4A shows schematically the microfluidic device used to measure the partition coefficients. Due to the constant slow flow, the concentrations in the test solution surrounding the gel plugs is maintained constant. The partition coefficient is given by the ratio of fluorescence intensity in the gel area compared to the test solution. Figures 4B-4D show the partition coefficients for the fluorescein dianion ${ }_{5}(10 \mu \mathrm{M})$ in $\mathrm{KCl}$ solutions of $1 \mathrm{mM}, 10 \mathrm{mM}, 100 \mathrm{mM}$ and $1 \mathrm{M}$ concentration, along with the three theoretical models (Donnan, solid nanochannel, best fit soft nanochannel for $\alpha=16$ ).

For different hydrogels, a power-law dependence between the pore size and the polymer concentration has typically been observed, although the numerical value of the exponent varies 10 somewhat (in the range of $-0.3^{5}$ to $-1.6^{29}$ ). The numerical values of the pore size, for similar concentrations, correspond approximately to values measured with other techniques for alginate gels covalently crosslinked with glutaraldehyde ${ }^{17}$, and also for ionically crosslinked alginate gels with low guluronic 15 acid content and thus low effective crosslinking density ${ }^{30}$. It should finally be said that the gels also exhibit substantial swelling at lower ionic force, such that we calculate the pore size at the relevant conditions by using the final total alginate concentration (details given in Electronic Supplementary 20 Information 3).

We then quantified the partition coefficient of the fluorescein dianion in gels of different alginate concentration $(25 \mathrm{mg} / \mathrm{mL}$, $50 \mathrm{mg} / \mathrm{mL}$, and $65 \mathrm{mg} / \mathrm{mL}$ ) at different ionic force $(1 \mathrm{mM}$ to $1 \mathrm{M}$ $\mathrm{KCl}$ ). To do so, we used a microfluidic setup with small disk ${ }_{25}$ shaped gel plugs in a microfluidic channel ${ }^{31}$ (Fig. 4A). This allows to maintain a constant small flow rate next to the gels, such as to precisely impose the composition of the bulk fluid surrounding the gels. For each measurement, we allowed at least $2 \mathrm{~h}$ of incubation with the desired bulk fluid. At this time, the 30 fluorescence intensity in the gel plugs had become homogeneous.

We then determined the partition coefficient by comparing the fluorescence intensity within the gel plugs to the fluorescence intensity in the bulk solution. Fig. $4 \mathrm{~B}$ to $4 \mathrm{D}$ show the experimental values of the partition coefficients for the different ${ }_{35}$ gel concentrations and ionic forces. We compared these values to the theoretically calculated values for the Gibbs-Donnan, solid nanochannel and soft hydrogel models, with matching pore sizes. It is obvious that the Gibbs-Donnan model predicts much too small partition coefficients (by up to 3 orders of magnitude), 40 indicating that the assumption of a spatially homogeneous space charged distribution in the hydrogels examined is clearly not valid. The solid nanochannel model provides a much better match. The soft hydrogel model, allowing for partial chain mobility, provides a further improvement only for the lowest 45 alginate concentration $(25 \mathrm{mg} / \mathrm{mL})$.

We also applied the Ogston model of pore size distribution ${ }^{5}$ to the solid nanochannel model (eq. 12). We find that this has very minor effect on the partition coefficient for the fluorescein dianion; if anything, it leads to a slight deterioration rather than 50 an improvement of the fit (details given in Electronic Supplementary Information 4).

Finally, we examined the partition of free, uncrosslinked alginate into the crosslinked gels. This allows to test whether the models developed for small ions are applicable to macromolecules as 55 well. Given that the non-crosslinked probe is otherwise chemically identical to the crosslinked gel, the measurement also allows to obtain an estimate of the electrostatic repulsion encountered by the alginate chains within the gel. The results are shown in Fig. 5. First, for all gel concentrations and ionic forces 60 examined, the Gibbs-Donnan model predicted much too low partition coefficients - the predictions are off by 10 to more than 100 orders of magnitude(!). This clearly confirms that for the partition of macromolecular substances, the local gel structure is very important and cannot simply by ignored. The solid 65 nanochannel model, assuming a network with homogeneous pore size, performs very well for the $25 \mathrm{mg} / \mathrm{mL}$ gels, but it predicts substantially too small partition coefficients for the higher alginate concentrations, namely for the lower ionic forces. Taking into account heterogeneous pore size distribution according to the ${ }_{70}$ Ogston $\operatorname{model}^{5}$ (eq. 12) improves the fit at higher alginate concentrations, although some overestimation results at $25 \mathrm{mg} / \mathrm{mL}$. The soft hydrogel model adapted to partition of highly charged macromolecular probes (eq. 11, for a best-fit value of $\beta=175$ ) performs reasonably well for all conditions. 


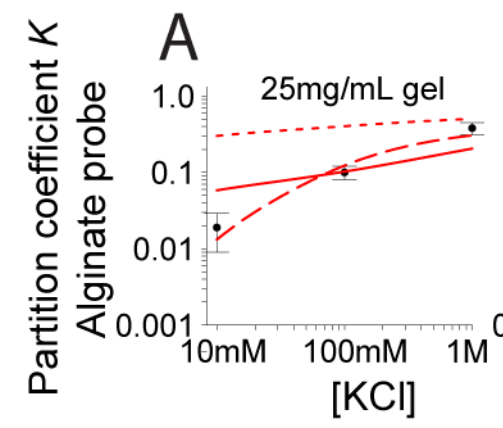

Gibbs-Donnan: $K=1.1^{\star} 10^{-45}$ to $2.8^{*} 10^{-11}$

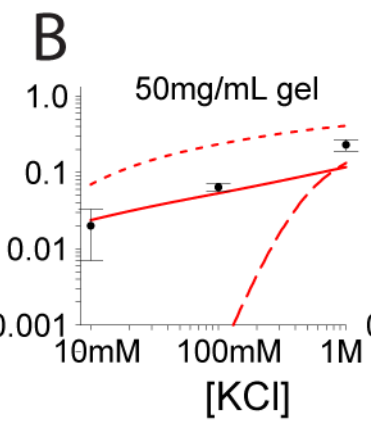

Gibbs-Donnan:

$K=2.9^{\star} 10^{-87}$ to $3.5^{*} 10^{-22}$
C

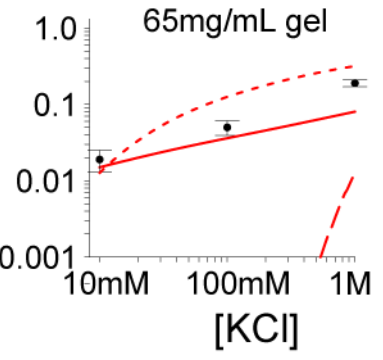

Gibbs-Donnan:

$K=4.2^{*} 10^{-111}$ to $2.8^{*} 10^{-31}$

\begin{tabular}{rlll}
\hline - Measured Models: & $-\cdots$ & solid nanochannel (Ogston) \\
& & & solid nanochannel (monodisperse) \\
& & soft hydrogel
\end{tabular}

Figure 5: Partition coefficients for free alginate 5A-5C show the partition coefficients for the free alginate tracer solutions in $\mathrm{KCl}$ of $10 \mathrm{mM}, 100 \mathrm{mM}$ and $1 \mathrm{M}$ concentration for three different alginate gel concentrations as indicated. Two variants of the solid nanochannel theory are shown, one assuming a monodisperse pore size, the other assuming a distribution of pore sizes as described by the Ogston model. We also indicate the predictions of the partition 5 coefficients for the soft hydrogel model (eq. 11) for the best fit parameter $\beta=175$, and the numerical values of the partition coefficients predicted by the Gibbs-Donnan equilibrium, too low to be shown on the same graphs. In the solid nanochannel model and the Gibbs-Donnan theory, molecular weight polydispersity and Manning condensation were explicitly taken into account, in the soft hydrogel model they are empirically accounted for by the best fit parameter $\beta$.

\section{Discussion}

${ }_{10}$ We investigate ionic partition in charged covalent alginate hydrogels in relation to the underlying nanostructure. We first quantified the partition of a small negatively charged probe, namely fluorescein dianion, into the alginate gels at different externally imposed ionic forces. As expected, we find that lower 15 ionic force and higher alginate concentration lead to more efficient ionic exclusion (Fig. 4). We compared the experimental results to three theoretical models for the local ionic distribution at the size scale of individual pores in the alginate hydrogels: the Gibbs-Donnan model, the solid nanochannel model, and the soft

20 hydrogel model (Fig. 2, Fig. 4). As in our conditions, the measured pore diameters always exceed the Debye length, large differences arise between the different models, and it is crucial to identify the correct model for the experimental conditions at hand.

${ }_{25}$ For the partition of the fluorescein dianion tracer, we find the soft hydrogel model to provide the best match, but especially for the higher gel concentrations, the solid nanochannel model provides a good match as well (Fig. 4). The Gibbs-Donnan model predicts generally much too low partition coefficients. This indicates that

30 the alginate chains are relatively immobile at the time scale of ionic redistribution, especially for the higher alginate concentrations. This is consistent with a high electrostatic repulsion between the alginate chains, namely for the higher alginate concentrations, as suggested by the Young modulus 35 measurement (Fig. 3). Indeed, the compressive Young modulus for the higher alginate concentrations $(50 \mathrm{mg} / \mathrm{mL}$ and $65 \mathrm{mg} / \mathrm{mL})$ shows a monotonous dependence on the ionic force, lower ionic force being associated with higher Young moduli (Fig. 3B). Given that the gels also swell more at lower ionic force (data in 40 Electronic Supplementary Information 3), this indicates higher Young moduli for higher swelling ratios for a given alginate concentration. Such an increase of Young modulus with increasing swelling is a relatively uncommon condition known to be related to a highly extended state of the hydrogel network ${ }^{32}$, 45 which we attribute here to dominating electrostatic repulsion between the gel strands. On the contrary, for the $25 \mathrm{mg} / \mathrm{mL}$ gel, a minimum Young modulus is found for $100 \mathrm{mM} \mathrm{KCl}$, with higher values both for higher and lower ionic force (Fig. 3B). This indicates a transition from a highly extended network to a so partially coiled state of the chains at higher ionic force ${ }^{32}$, a finding consistent with the more important role of chain mobility for the partition of fluorescein dianion into the $25 \mathrm{mg} / \mathrm{mL}$ gels.

The good fit between the solid nanochannel model and the observed partition coefficients for the $50 \mathrm{mg} / \mathrm{mL}$ and $65 \mathrm{mg} / \mathrm{mL}$ ${ }_{55}$ gels (Fig. 4) indicates that for these harder gels, the hydraulic pore diameter is also the relevant pore diameter for the partition of small ions. The hydraulic pore diameter is therefore analogous to the physical pore dimensions in manmade nanochannels ${ }^{7,27}$, or the intermolecular distance in polyelectrolyte solutions ${ }^{12}$. This 60 analogy allows to directly transpose conceptual and mathematical developments made in these domains to the charged hydrogels. Namely, deviations of polyelectrolyte solutions from the GibbsDonnan equilibrium are very well studied ${ }^{12,13,33}$, and the analytical expression for the partition coefficient in the solid ${ }_{65}$ nanochannel model presented here (eq. 10) is based on these developments $^{12,33}$

A further important point is that the rough approximation by a 1D lattice allows to model quantitatively the ion exclusion in the 3D hydrogels. Analogous observations have been made before for 70 polyelectrolyte solutions ${ }^{33}$; it is commonly assumed that this reflects local lattice-like self organization due to electrostatic repulsion between the chains ${ }^{12}$, leading to homogeneous spacing between the chains in all space directions. This view is backed by our data on the mechanical and swelling properties, as they 75 indicate an extended chain configuration associated with strong interchain electrostatic repulsion; it is further corroborated by the 
experimentally observed optical clarity of the covalent alginate hydrogels, as this indicates absence of structural inhomogeneity beyond the $100 \mathrm{~nm}$ scale ${ }^{34}$.

For the partition of the macromolecular alginate probe, we find 5 that the solid nanochannel model with a monodisperse pore size, and even more so the Gibbs-Donnan model predict partition coefficients orders of magnitude below the observed ones (Fig. 5). For the partition of macromolecules into hydrogels, it is generally assumed that pore size variation needs to be taken into 10 account, as described by the Ogston model ${ }^{5}$. Indeed, we find the fit to be improved by allowing for a pore size distribution according to the Ogston model ${ }^{5}$, rather than a single homogeneous pore size (Fig. 5, Ogston model). This contrasts with the results obtained for the low molecular weight probe

15 fluorescein dianion, since applying the Ogston model to this probe yields no improvement (Electronic Supplementary Information 4). This most likely implies that the highly charged free alginate probe is able to locally deform the gel, such as to create the Ogston pore structure transiently in response to the ${ }_{20}$ presence of the probe molecules. This is also suggested by the very good fit between the soft hydrogel model and the experimental data for the alginate tracer (Fig. 5), since the underlying assumption of the soft hydrogel model for the high molecular weight tracer is that the probe is able to make room for 25 itself and ultimately has access to a volume fraction determined by electrostatic repulsion from the crosslinked chains much as if it were part of the network itself. A dynamic pore structure due to charge interactions might also explain the peculiar observation, made by $\mathrm{us}^{25}$ and others ${ }^{21}$, that diffusion coefficients of 30 macromolecules in charged gels are much less affected by ionic force than the partition coefficients.

The theoretical and experimental results presented in this report place important conditions on the use of different models to describe molecular partition in charged hydrogels. The Gibbs-

35 Donnan model greatly overestimates the efficiency of co-ion exclusion from the charged hydrogels, the discrepancy to experimental results being inacceptable namely for the macromolecular probe. Taking into account the nanoporous structure in the solid nanochannel model substantially improves 40 the fit between theory and observed data. However, we globally find that the best match between theory and observed partition coefficients is obtained by the soft hydrogel model, for both the low molecular weight (eq. 6-C) and high molecular weight version (eq. 11). Interestingly, the numerical simulation indicates 45 that the Gibbs-Donnan model, frequently used for its simplicity and elegance, is appropriate for the partition of the counter-ions, making up the majority of the mobile ionic species in charged hydrogels, even if the condition of a small pore size as compared to the Debye length is not met (Fig. 2E). This asymmetry ${ }_{50}$ between the distribution of co- and counter-ions is known to be associated with high fixed local space charge, ${ }^{12}$ and it implies that it may be suitable to use the Gibbs-Donnan model for properties related to majority ionic species, under conditions not necessarily compatible with the basic assumption of homogeneous space

${ }_{55}$ charge distribution. For instance, the osmotic pressure within the gels is a crucial element in the understanding of hydrogel swelling within the Flory-Huggins theory ${ }^{17}$, where it is successfully evaluated using the Gibbs-Donnan model modified for counter-ion condensation, but only with minor activity 60 coefficient corrections ${ }^{17}$.

In terms of scaling laws, the ratio $\lambda$ between nominal pore size and the Debye length (eq. 3 ) is known to be of prime importance in the understanding of charged hydrogels; ${ }^{15}$ indeed, it reflects the mean spacing between polymer strands as compared to the Debye ${ }_{65}$ length. It is well established that if this ratio is below unity, a nearly exact match between the Gibbs-Donnan model and models taking into account that nanostructure can be expected both for co- and counter-ions ${ }^{15}$. We confirm this by numerical simulation in Fig. 2E. For larger values of $\lambda$, we find that it is mandatory to 70 take local nanostructure into account for co-ion exclusion, although not necessarily for counter-ion enrichment (Fig. 2E). In addition, we also find $\lambda$ to dictate the effective charge distribution relevant for the low molecular weight probe in the soft hydrogel model (equation 6-C). Finally, by means of equation 11, we 75 provide a direct link between $\lambda$ and the partition of the highly charged macromolecular probe.

\section{Experimental}

The following chemicals were obtained from Sigma-Aldrich (Buchs, Switzerland): Alginate sodium salt (catalog number ${ }_{80}$ A0682), morpholinoethanesulfonic acid (MES, catalog number M2933), adipic acid dihydrazide (AAD, A0638), N-(3dimethylaminopropyl)-N'-ethylcarbodiimide hydrochloride (EDC, E7750), sodium hydroxide (S8045), aminofluorescein (07980), potassium chloride (P9541), fluorescein disodium 85 salt(F6377); isopropanol (99295) was obtained from Reactolab (Servion, Switzerland).

Covalent alginate hydrogels were synthesized by established carbodiimide chemistry ${ }^{24,25}$. Briefly, the different gels were made as follows: gel-1 $(25 \mathrm{mg} / \mathrm{mL}): 1 \mathrm{~mL} 30 \mathrm{mg} / \mathrm{mL}$ alginate, $0.17 \mathrm{~mL}$ 90 MES $\mathrm{pH} 5.5,0.5 \mathrm{M}, 25 \mu \mathrm{L}$ adipic acid dihydrazide $50 \mathrm{mg} / \mathrm{mL}$, EDC $12 \mathrm{mg}$ in $12 \mu \mathrm{L} \mathrm{DI}$; gel-2 $(50 \mathrm{mg} / \mathrm{mL}): 1 \mathrm{~mL} 60 \mathrm{mg} / \mathrm{mL}$ alginate, $0.2 \mathrm{~mL}$ MES $\mathrm{pH} 5.5,0.5 \mathrm{M}, 50 \mu \mathrm{L}$ adipic acid dihydrazide $50 \mathrm{mg} / \mathrm{mL}$, EDC $24 \mathrm{mg}$ in $24 \mu \mathrm{L}$ DI; gel-3 $(65 \mathrm{mg} / \mathrm{mL}): 1 \mathrm{~mL} 90 \mathrm{mg} / \mathrm{mL}$ alginate, $0.2 \mathrm{~mL}$ MES $\mathrm{pH} 5.5,0.5 \mathrm{M}$, ${ }_{95} 75 \mu \mathrm{L}$ adipic acid dihydrazide $50 \mathrm{mg} / \mathrm{mL}$, EDC $36 \mathrm{mg}$ in $36 \mu \mathrm{L}$ DI. Gel formation was initiated within 2 minutes of mixing at room temperature, but let to complete for at least $1 \mathrm{~h}$ prior to gel use. Mechanical testing was performed on a textureAnalyzer (stable MicroSystems), using hydrogel disks of $7 \mathrm{~mm}$ diameter 100 and $1 \mathrm{~mm}$ height. Prior to compression, the gel disks were equilibrated in the desired buffer solution ( $\mathrm{KCl}$ or $\mathrm{PBS}$ ), and compression was performed with the disks submerged in the buffer solution. The gels were compressed by $20 \%$ at a rate of $30 \mu \mathrm{m} / \mathrm{s}$, and the resulting force recorded. Once the final 105 compression achieved, stress relaxation was monitored at constant compression by continuously recording the slowly decreasing elastic force. Strains were calculated by the deformation relative to the original height, and stress as the force per area. Partition coefficients of fluorescein dianion and 110 fluorescently labeled alginate were measured by quantification of fluorescence intensity in the gel and in surrounding perfusion medium of the desired composition. This was carried out in a microfluidic channel, comprising a gel plug and a perfusion solution flowing around the gel plug in the channel. The 115 microfluidic channels themselves were molded in PDMS with a 
channel height of $50 \mu \mathrm{m}$, and the gel plugs produced by deposition of a drop of the fully mixed, yet still liquid gel precursor solution on to the PDMS before pressing it against a microscope slide. Once the gel plug polymerized, excess EDC 5 and soluble reaction products were washed extensively by flushing PBS, and then DI through the channels. To evaluate the partition coefficient of non-crosslinked alginate, we synthesized fluorescently labeled alginate according a previously reported protocol, with modification $^{35}: 4 \mathrm{~g}$ of alginate were dissolved in 10 200mL MES pH 5.5, 50mM. Next, 200mg of aminofluorescein were dissolved in $2 \mathrm{~mL} \mathrm{NaOH} 1 \mathrm{M}$, and the solution stirred into the alginate solution. $40 \mathrm{mg}$ of EDC, dissolved in $1 \mathrm{~mL}$ of MES $\mathrm{pH} 5.5,50 \mathrm{mM}$ was added next, and the solution stirred. After $1 \mathrm{~h}$ of reaction time at room temperature, the alginate was

15 precipitated in an excess of isopropanol (1L), and further washed with $80 \%$ isopropanol $/ 20 \%$ deionized water, and then isopropanol washes until the supernatant remained visually free of aminofluorescein (i.e. without any perceptible yellow hue). Most isopropanol was then squeezed out of the precipitate, and the 20 alginate redissolved in deionized water. The precipitation and isopropanol washing procedure was then repeated once more to remove traces of remaining free aminofluorescein. To obtain dry material, the alginate was extensively dried in an oven at $40^{\circ} \mathrm{C}$. The molecular weight distribution of both the starting material 25 and the labeled samples were obtained by Gel Permeation Chromatography (GPC 50+ Agilent, eluent NaNO3 10mM + $10 \% \mathrm{MeOH}$, PL aquagel-OH MIXED-M; starting material: $\mathrm{M}_{\mathrm{n}}=110.1 \mathrm{kDa}, \quad \mathrm{M}_{\mathrm{w}}=137.9 \mathrm{kDa}, \quad \mathrm{PD}=1.44$, labeled sample: $\mathrm{M}_{\mathrm{n}}=94.8 \mathrm{kDa}, \mathrm{M}_{\mathrm{w}}=147.8 \mathrm{kDa}, \mathrm{PD}=1.56$, detailed distribution 30 curves in Electronic Supplementary Information 1). Numerical solution of the Poisson-Boltzmann equation (eq. 1) under suitable boundary conditions (eq. 2) and fixed charge distribution (eq. 5, eq. 6-A, 6-B, or 6-C) was performed in the free software R-cran, by means of a custom package available for download in the

35 Electronic Supplementary Information. Installation and usage instructions for this package are provided as Electronic Supplementary Information 5; an implementation to evaluate eq. 10 is also supplied in this package (see Electronic Supplementary Information 5 and 6).

\section{${ }_{40}$ Conclusions}

We investigate the ionic partition of negatively charged co-ions into negatively charged covalent alginate hydrogels, at ionic strengths ranging from $1 \mathrm{mM}$ to $1 \mathrm{M} \mathrm{KCl}$. We find that the GibbsDonnan model is not appropriate for the partition of the co-ions, ${ }_{45}$ due to a pore size substantially exceeding the Debye length. This makes the use of a Poisson-Boltzmann model necessary. We further find that while taking into account hydrogel polymer strand mobility affords limited improvement in the prediction of the partition coefficients of the low molecular weight tracer 50 fluorescein dianion, it is essential to take into account gel deformation in response to macromolecular probes. This can be done with substantial numerical effort by combining PoissonBoltzmann simulations with heterogeneous pore size distribution as suggested by the Ogston model, or more simply, by applying a 55 semi-empirical scaling law analysis of electrostatic repulsion in terms of the ratio between Debye length and pore size.

In terms of drug delivery and chromatographic applications, the flexible nanofluidics encountered in the covalent alginate hydrogels imply that the range of molecules that can be loaded is ${ }_{60}$ greater than what one would expect, certainly as based on the Gibbs-Donnan theory. Namely, even high molecular weight, highly like-charged molecules show an appreciable partition coefficient at physiological ionic force.

Finally, the effects of strand mobility provide a conceptual ${ }_{65}$ bridge between the Poisson-Boltzmann framework appropriate for the small probes, and the Ogston framework for taking into account pore size variation for larger probes.

\section{Acknowledgements}

We wish to acknowledge Dr. Arnaud Bertsch for proofreading 70 the manuscript, and the EPFL Molecular and Hybrid Materials Characterization Center for carrying out the GPC analysis. Funding was provided by the Swiss National Science Foundation, Grant PBELP3-133350.

\section{Notes and references}

$75 \dagger$ Electronic Supplementary Information (ESI) available:

Electronic Supplementary Information 1 gives the detailed procedure to theoretically estimate the partition coefficient of macromolecular species Electronic Supplementary Information 2 outlines the theoretical development of the relation between stress relaxation and pore size (eq. 8012 )

Electronic Supplementary Information 3 gives experimental and theoretical details on alginate hydrogel swelling

Electronic Supplementary Information 4 illustrates the prediction of the Ogston model for the partition of fluorescein dianion.

85 Electronic Supplementary Information 5 provides installation and usage instructions for the custom code used to solve numerically the PoissonBoltzmann equation. The custom code itself is available as an R-package (poisson.boltzmann.1D.tar.gz for MacOSX and Linux, and poisson.boltzmann.1D.zip for windows).

90 Electronic Supplementary Information 6 provides analytical expressions approximating the Poisson-Boltzmann solution

\section{See DOI: $10.1039 / \mathrm{b} 000000 \mathrm{x} /$}

95 1. O. Lieleg and K. Ribbeck, Trends Cell Biol, 2011, 21, 543-551.

2. G. H. Lathe and C. R. J. Ruthven, Biochemical Journal, 1956, 62, 665-674.

3. T. R. Hoare and D. S. Kohane, Polymer, 2008, 49, 1993-2007.

4. S. Raymond and L. Weintraub, Science, 1959, 130, 711-711.

100 5. D. Rodbard and A. Chrambach, Proc Natl Acad Sci U S A, 1970, 65, 970-977.

6. G. Sadowski, W. Richtering, J. Höpfner, T. Richter, P. Kosovan, C. Holm and M. Wilhelm, in Intelligent Hydrogels, Springer International Publishing, 2013, pp. 247-263.

105 7. A. Plecis, R. B. Schoch and P. Renaud, Nano Lett, 2005, 5, 1147 1155

8. A. L. Garcia, L. K. Ista, D. N. Petsev, M. J. O'Brien, P. Bisong, A. A. Mammoli, S. R. J. Brueck and G. P. Lopez, Lab on a Chip, 2005, 5, 1271-1276.

110 9. A. Yaroshchuk, O. Zhukova, M. Ulbricht and V. Ribitsch, Langmuir, 2005, 21, 6872-6882.

10. D. Chapman, The London, Edinburgh, and Dublin Philosophical Magazine and Journal of Science, 1913, 25, 475-481.

11. G. Gouy, Comptes Rendus de l'Académie des sciences, 1909, 149, 654-657. 
12. J. T. Overbeek, Prog Biophys Biophys Chem, 1956, 6, 57-84.

13. G. S. Manning, Chem. Phys., 1969, 51, 924.

14. F. G. Donnan, Zeitschrift für Elektrochemie und angewandte physikalische Chemie, 1911, 17, 572-581.

5 15. P. J. Basser and A. J. Grodzinsky, Biophys Chem, 1993, 46, 57-68.

16. J. Wang, in The nanoporous morphology of photopolymerized crosslinked polyacrylamide hydrogels, PhD Thesis at Texas A\&M University, 2008.

17. A. W. Chan and R. J. Neufeld, Biomaterials, 2009, 30, 6119-6129.

10 18. P. Banerjee, D. Lenz, J. P. Robinson, J. L. Rickus and A. K. Bhunia, Lab Invest, 2008, 88, 196-206.

19. A. Pluen, P. A. Netti, R. K. Jain and D. A. Berk, Biophys J, 1999, 77, 542-552.

20. J. S. Varghese, N. Chellappa and N. N. Fathima, Colloids Surf B Biointerfaces, 2014, 113, 346-351.

21. E. M. Johnson, in Partitioning and Diffusion of Macromolecules in Charged Gels, PhD thesis at Massachusetts Institute of Technology, Cambridge, 1995.

22. J. W. Moore and D. O. Hummel, Physikalische Chemie, deGruyter, $20 \quad$ Berlin, 1986.

23. P. Debye and E. Hückel, Physikalische Zeitschrift, 1923, 24, 185206.

24. X. Zhao, N. Huebsch, D. J. Mooney and Z. Suo, J Appl Phys, 2010, 107, 63509.

25 25. S. Wu, T. Braschler, R. Anker, F. Wildhaber, A. Bertsch, J. Bruegger and P. Renaud, Journal of Membrane Science, 2015, 477, 151156.

26. C. L. Chiu, V. Hecht, H. Duong, B. Wu and B. Tawil, Biores Open Access, 2012, 1, 34-40.

30 27. A. Plecis, R. B. Schoch and P. Renaud, in Microtas, Boston, 2005.

28. H. Darcy, Les fontaines publiques de la ville de Dijon, Librairie des corps impériaux des ponts et chaussées et des mines, Paris, 1856.

29. J. Narayanan, J. Y. Xiong and X. Y. Liu, Journal of Physics:

35 Conference Series, 2006, 28, 83-86.

30. J. Klein, J. Stock and K. D. Vorlop, Eur J Appl Microbiol, 1983, 18, 86-91.

31. D. Hlushkou, R. Dhopeshwarkar, R. M. Crooks and U. Tallarek, Lab Chip, 2008, 8, 1153-1162.

40 32. O. Okay, in Hydrogel Sensors and Actuators, eds. G. Gerlach and K.F. Arndt, Springer, Heidelberg, 2009.

33. F. W. Klaarenbeek, in Over Donnan-evenwichten bij solen van arabische gom, PhD Thesis at Universiteit Utrecht, 1946.

34. J. McCartney, Optics of the Atmosphere: Scattering by Molecules $45 \quad$ and Particles, Wiley, 1976.

35. T. Braschler, A. Valero, L. Colella, K. Pataky, J. Brugger and P. Renaud, Lab Chip, 2010, 10, 2771-2777. 